\title{
Identification of Optimal Autoregressive Integrated Moving Average Model on Temperature Data
}

Olusola Samuel Makinde

Federal University of Technology, osmakinde@futa.edu.ng

Olusoga Akin Fasoranbaku

Federal University of Technology, olusogaf@yahoo.com

Follow this and additional works at: http://digitalcommons.wayne.edu/jmasm

Part of the Applied Statistics Commons, Social and Behavioral Sciences Commons, and the Statistical Theory Commons

\section{Recommended Citation}

Makinde, Olusola Samuel and Fasoranbaku, Olusoga Akin (2011) "Identification of Optimal Autoregressive Integrated Moving Average Model on Temperature Data," Journal of Modern Applied Statistical Methods: Vol. 10 : Iss. 2, Article 31.

DOI: $10.22237 /$ jmasm/1320121800

Available at: http://digitalcommons.wayne.edu/jmasm/vol10/iss2/31

This Emerging Scholar is brought to you for free and open access by the Open Access Journals at DigitalCommons@WayneState. It has been accepted for inclusion in Journal of Modern Applied Statistical Methods by an authorized editor of DigitalCommons@WayneState. 


\title{
Identification of Optimal Autoregressive Integrated Moving Average Model on Temperature Data
}

\author{
Olusola Samuel Makinde Olusoga Akin Fasoranbaku \\ Federal University of Technology, \\ Akure, Nigeria
}

Autoregressive Integrated Moving Average (ARIMA) processes of various orders are presented to identify an optimal model from a class of models. Parameters of the models are estimated using an Ordinary Least Square (OLS) approach. $\operatorname{ARIMA}(p, d, q)$ is formulated for maximum daily temperature data in Ondo and Zaira from January 1995 to November 2005. The choice of ARIMA models of orders $p$ and $q$ is intended to retain persistence in a natural process. To determine the performance of models, Normalized Bayesian Information Criterion is adopted. The ARIMA $(1,1,1)$ is adequate for modeling maximum daily temperature in Ondo and Zaira; model parameters are estimated and redundant variables are removed. Causality and the invertibility behavior of some optimal models are also presented.

Key words: Autoregressive Integrated Moving Average, optimal, causality, invertibility, redundancy.

\section{Introduction}

A time series of $\mathrm{T}$ successive observations is regarded as a sample from an infinite population of a time-series that could have been generated by the stochastic process under study. A powerful way to extract useful information on the underlying process - solely on the basis of the past behavior of the time series itself - is the univariate Box-Jenkins approach. Although originally developed for forecasting purposes (Box \& Jenkins, 1976; Nelson, 1976), BoxJenkins models are useful tools for describing the time dependent structure of stationary and non-stationary time series. Box-Jenkins models for stationary time series, or ARIMA models, have been applied in many areas of research, for example in tree-ring chronologies (Meko, 1981), in the evolution of the unemployment rate (Dobre \& Alexandru, 2008), and in the analysis of UK Pounds/US Dollar exchange rate (Shittu and Yaya, 2009).

O. S. Makinde is Graduate/Research Assistant in the Department of Mathematical Sciences. Email him at: osmakinde@futa.edu.ng. O. A. Fasoranbaku is Senior Lecturer in the Department of Mathematical Sciences. Email him at: olusogaf@yahoo.com.
Models are initialized using observed data. As proposed by Lorenz (1963), long range forecasts - those made at a range of two weeks or more - are impossible to definitively predict the state of the atmosphere owing to the chaotic nature of the mechanism involved. Forecast models are used to determine future conditions. However, in real life research and practice, patterns of data are unclear and individual observations involve considerable error; thus, it is necessary to not only uncover the hidden patterns in the data but also to forecast. The ARIMA methodology (Box \& Jenkins, 1976) provides a method to accomplish these tasks.

Considering estimates of times series model parameters, Pham-Dinh (1978) computed the exact log likelihood of a time series model and also proposed and justified an asymptotic approximation of the model. Bobba, et al (2006) formulated a stochastic model simulating trends in hydrological and meteorological variables: Their choice of ARIMA model of orders $p$ and $q$ was intended to retain any persistence in the natural processes and they claimed that an ARIMA (1, 0, 1) model was adequate for modeling three variables of temperature, precipitation and stream flow on a seasonal basis in the North East Pond River Watershed. Ojo (2009) compared subsets of autoregressive integrated moving average models to full 
autoregressive integrated moving average models. The parameters of these models were estimated and the statistical properties of the derived estimates were investigated. In his study, he showed that subset autoregressive integrated moving average models performed better than full autoregressive integrated moving average models. Makinde (2011) investigated the behavioural pattern of invertibility parameter $\pi_{i}$ of the ARIMA (p, d, q) model for various $p$ and $d$. He showed that behaviour of $\pi_{i}$ depends on the order of autoregressive part $(p)$, the order of integrated part $(d)$, positive and negative values of moving average parameter $(\theta)$. Similarly, Fasoranbaku \& Makinde (2011) investigated causality parameter of ARMA model. From their findings, It is deduced that the behaviour of causality parameter $\psi_{k}$ depends on positive and negative values of autoregressive parameter $\phi$ and moving average parameter 9 .

In this study, we shall evaluate parameters of $\operatorname{ARIMA}(p, d, q)$ for various values of $p$ and $d$ using an ordinary least squares (OLS) method and Crammer's rule; identify optimal model in a class of ARIMA models for temperature profile of two cities in Nigeria and check for redundant variables in the models using a t-test.

\section{Stationarity and Test of Stationarity}

A process is said to be strictly stationary if, for any value of $j_{1}, j_{2}, \ldots, j_{n}$, the joint distribution of $\left(y_{t}, y_{t+1}, y_{t+2}, \ldots, y_{t+j}\right)$ depends only on the interval separating the dates $\left(j_{1}, j_{2}, \ldots, j_{n}\right)$, and not on the date $(t)$ itself. If a process is strictly stationary with finite second moments, then it must be covariance stationary (Hamilton, 1994).

In short, if a time series is stationary, its mean, variance, and autocovariance (at various lags) remain the same regardless of the point at which they are measured; that is, they are time invariant. There are several tests of stationarity; which include: (1) graphical analysis, (2) a correlogram, and (3) unit root test, e.t.c. For a stationary time series, a correlogram tapers quickly; whereas for non-stationary time series it dies off gradually. If autocorrelations start high and decline slowly, then the series is nonstationary and should be differenced. Similarly, an ARIMA process is said to be stationary if spikes decay to zero after a few lags. In this study, correlogram use was adopted to test for stationarity of temperature data.

Test for Model Adequacy

To test the adequacy of the model, the Ljung-Box (1978) statistic will be used; this is a statistical test for determining whether any of a group of autocorrelations of a time series is different from zero. As opposed to testing randomness at each distinct lag, it tests the overall randomness based on a number of lags, and is therefore a portmanteau test. The LjungBox Statistic is:

$$
Q(\hat{r})=n(n+2) \sum_{k=1}^{h} \frac{\hat{r}_{k}}{n-k} .
$$

Specification of ARIMA in Terms of A Lag Operator

When the models are specified in terms of the lag operator L, the AR (p) model is given by

$$
\varepsilon_{t}=\left(1-\sum_{i=1}^{p} \phi_{i} L^{i}\right) y_{t}=\phi(L) y_{t},
$$

where

$$
\phi(L)=1-\sum_{i=1}^{p} \phi_{i} L^{i}
$$

and the MA(q) model is given by

$$
y_{t}=\left(1+\sum_{i=1}^{p} \theta_{i} L^{i}\right) \varepsilon_{t}=\theta(L) \varepsilon_{t},
$$

where

$$
\theta(L)=1+\sum_{i=1}^{p} \theta_{i} L^{i}
$$

$\operatorname{ARIMA}(p, 0, q)$ is

$$
\left(1-\sum_{i=1}^{p} \phi_{i} L^{i}\right) y_{t}=\left(1+\sum_{i=1}^{p} \theta_{i} L^{i}\right) \varepsilon_{t}
$$




\section{IDENTIFICATION OF OPTIMAL ARIMA MODEL ON TEMPERATURE DATA}

or more concisely:

$$
\phi(L) y_{t}=\theta(L) \varepsilon_{t}
$$

which implies $y_{t}=\psi(L) \varepsilon_{t}$, where

$$
\begin{aligned}
\psi(L) & =\frac{\theta(L)}{\phi(L)} \\
& =\frac{1+\theta_{1} L+\theta_{2} L^{2}+\ldots+\theta_{p} L^{p}}{1-\phi_{1} L-\phi_{2} L^{2}-\ldots-\phi_{p} L^{p}} .
\end{aligned}
$$

The ARIMA process is stationary if This occurs if the series $\psi(Z)$ converges for every $Z$ with $|Z| \leq 1$. Because $\psi(Z)$ is a rational function, the series converges for every $Z$ with $|Z| \leq 1$ if the complex zeros of $\phi(Z)$ lie outside the unit circle. If a process is stationary, then because $\mathrm{y}_{\mathrm{t}}=\psi(\mathrm{L}) \varepsilon_{\mathrm{t}}$, and the expected values of $\varepsilon_{t}$ are all 0 , the expected value of $y_{t}$ is also 0 .

\section{Causality of Some ARIMA Processes}

Some ARIMA processes of various orders are shown in causal form to provide a useful way of generating a random sequence. That is, a linear process $y_{t}$, as a linear combination of white noise variates $\varepsilon_{t}$. For an ARIMA $\quad(1, \quad 0, \quad 1) \quad$ process, $y_{t}=c+\phi y_{t-1}+\varepsilon_{t}+\theta \varepsilon_{t-1} \quad$ implies that $y_{t}=(1-\phi L)^{-1} c+(1-\phi L)^{-1}\left(\varepsilon_{t}+\theta \varepsilon_{t-1}\right) \quad$ which gives

$$
y_{t}=\frac{c}{(1-\phi)}+\varepsilon_{t}+\sum_{i=1}^{\infty}\left(\phi^{i}+\theta \phi^{i-1}\right) \varepsilon_{t-i},
$$

where $i=1,2, \ldots$ with $E\left(y_{t}\right)=\mu=\frac{c}{(1-\phi)}$; this holds only if $\phi \neq 1$.

For an ARIMA $(1,0,2)$ process, $y_{t}=c+\phi y_{t-1}+\varepsilon_{t}+\theta_{1} \varepsilon_{t-1}+\theta_{2} \varepsilon_{t-2}$ which gives

$$
\begin{gathered}
y_{t}=\frac{c}{(1-\phi)}+\varepsilon_{t}+\sum_{i=1}^{\infty}\left(\phi^{i}+\theta_{1} \phi^{i-1}+\theta_{2} \phi^{i-2}\right) \varepsilon_{t-i} \\
y_{t}=\frac{c}{(1-\phi)}+\sum_{i=0}^{\infty} \psi_{i} \varepsilon_{t-i}
\end{gathered}
$$

where $\quad \psi_{0}=1, \quad \psi_{1}=\phi+\theta \quad$ and $\psi_{j}=\phi^{j}+\theta_{1} \phi^{j-1}+\theta_{2} \phi^{j-2}, j=2,3,4, \ldots$; this is valid if $\phi \neq 1$.

Fasoranbaku \& Makinde (2011) has shown that the causality parameter $\psi_{i}$ is skewed to the right and sinusoidal for positive and negative values of $\phi$ respectively. Absolute value of causality parameter $\psi_{i}$ of ARIMA $(1,0, q)$ increases as the value of $q$ increases for positive values of $\phi$. The behavioural pattern of the causality parameters for $d=0$ and $\|\phi\|<1$ is well studied in Fasoranbaku \& Makinde (2011).

Representation ARIMA Models in Inverted Form

An ARIMA $(p, d, q)$ process is said to be invertible if the series converges in mean to $\varepsilon_{z}$ as $p \rightarrow \infty$. This happens when $\theta(Z)-0$ lie outside the unit circle. An ARIMA $(p, d, q)$ process is invertible if the absolute value of the parameters of ARIMA $(p, d, q)$ model satisfy $\left|\theta_{i}\right|<1$ for $i=1, \ldots, q$.

$\operatorname{ARIMA}(1,0,1)$

$$
\begin{gathered}
y_{t}=c+\phi y_{t-1}+\varepsilon_{t}+\theta \varepsilon_{t-1} \\
\varepsilon_{t}=-\frac{c}{(1+\theta)}+y_{t}+\sum_{i=1}^{\infty}(-1)^{i}\left(\theta^{i}+\phi \theta^{i-1}\right) y_{t-i} \\
y_{t}=\frac{c}{(1+\theta)}+\sum_{i=1}^{\infty}(-1)^{i+1}\left[\phi \theta^{i-1}+\theta^{i}\right] y_{t-i}+\varepsilon_{t}
\end{gathered}
$$

$$
y_{t}=\frac{c}{(1+\theta)}+\sum_{i=1}^{\infty} \pi_{i} y_{t-i}+\varepsilon_{t},
$$

where $\quad \pi_{i}=(-1)^{i+1}\left[\phi \theta^{i-1}+\theta^{i}\right], i=1,2,3, \ldots ;$ this holds if $\theta \neq-1$. 
ARIMA $(1,1,1)$

$$
\begin{gathered}
\Delta y_{t}=c+\phi \Delta y_{t-1}+\varepsilon_{t}+\theta \varepsilon_{t-1} \\
s_{t}=-\frac{c}{(1+\theta)}+\sum_{i=0}^{\infty} \pi_{i} y_{t-i} \\
\pi_{i}=\left\{\begin{array}{cc}
1, & i=0 \\
-(\theta+\phi+1), & i=1 \\
(-1)^{i}\left[(\theta+\phi)\left(\theta^{i-1}+\theta^{i-2}\right)\right], i=2, \ldots
\end{array}\right.
\end{gathered}
$$

$\operatorname{ARIMA}(2,0,1)$

$$
\begin{gathered}
y_{t}=c+\phi_{1} y_{t-1}+\phi_{2} y_{t-2}+\varepsilon_{t}+\theta \varepsilon_{t-1}, \\
y_{t}=\left[\begin{array}{l}
\frac{c}{(1+\theta)}+\left(\theta+\phi_{1}\right) y_{t-1} \\
+\sum_{i=2}^{\infty}(-1)^{i+1}\left[\theta^{i}+\phi_{1} \theta^{i-1}\right. \\
\left.+(-1)^{i+1} \phi_{2} \theta^{i-2}\right] y_{t-i}+\varepsilon_{t}
\end{array}\right], \\
y_{t}=\frac{c}{(1+\theta)}+\sum_{i=1}^{\infty} \pi_{i} y_{t-i}+\varepsilon_{t},
\end{gathered}
$$

where $\pi_{1}=\phi_{1}+\theta$ and

$$
\pi_{i}=(-1)^{j+1}\left[\theta^{j}+\phi_{1} \theta^{j-1}+(-1)^{j+1} \phi_{2} \theta^{j-2}\right],
$$

$j=2,3,4, \ldots$; this holds if $\theta \neq-1$.

Makinde (2011) has shown that invertibility parameter $\pi_{i}$ of ARIMA $(p, d, 1)$ for various integer values of $d$ are sinusoidal, the absolute value of the invertibility parameter, $\left|\pi_{i}\right|$ increases as $d$ increases for positive values of $\theta$ and the lower the integer value of $d$, the faster $\left|\pi_{i}\right|$ converges to zero. The behavioural pattern of the invertibility parameter is well discussed in Makinde (2011).

Evaluation of ARIMA $(p, d, q)$

$\operatorname{ARIMA}(1,0,0)$

$$
y_{t}=c+\phi y_{t-1}+\varepsilon_{t}
$$

If $c=0$, then $\sum_{t=1}^{T} y_{t} y_{t-1}-\phi \sum_{t=1}^{T} y_{t-1}^{2}=0$, which implies that

$$
\hat{\phi}=\frac{\sum_{t=1}^{T} y_{t} y_{t-1}}{\sum_{t=1}^{T} y_{t-1}^{2}} .
$$

If $c \neq 0$, equation (8) gives

$$
\left(\begin{array}{cc}
n & \sum_{t=1}^{T} y_{t-1} \\
\sum_{t=1}^{T} y_{t-1} & \sum_{t=1}^{T} y_{t-1}^{2}
\end{array}\right)\left(\begin{array}{l}
c \\
\phi
\end{array}\right)=\left(\begin{array}{c}
\sum_{t=1}^{T} y_{t} \\
\sum_{t=1}^{T} y_{t} y_{t-1}
\end{array}\right)
$$

Using Crammer's rule to solve for $c$ and $\phi$ results in

$$
\hat{c}=\frac{\nabla_{1}}{\nabla} \text { and } \hat{\phi}=\frac{\nabla_{2}}{\nabla}
$$

where

$$
\begin{gathered}
\nabla=n \sum_{t=1}^{T} y_{t-1}^{2}-\left[\sum_{t=1}^{T} y_{t-1}\right]^{2} \\
\nabla_{1}=\left[\sum_{t=1}^{T} y_{t}\right]\left[\sum_{t=1}^{T} y_{t-1}^{2}\right]-\left[\sum_{t=1}^{T} y_{t-1}\right]\left[\sum_{t=1}^{T} y_{t} y_{t-1}\right]
\end{gathered}
$$

and

$$
\nabla_{2}=n \sum_{t=1}^{T} y_{t} y_{t-1}-\left[\sum_{t=1}^{T} y_{t}\right]\left[\sum_{t=1}^{T} y_{t-1}\right] .
$$

$\operatorname{ARIMA}(1,1,0)$

$$
\Delta y_{t}=c+\phi \Delta y_{t-1}+\varepsilon_{t}
$$

when $c=0, \Delta y_{t}=\phi \Delta y_{t-1}+\varepsilon_{t}$ which gives

$$
\begin{gathered}
y_{t}-y_{t-1}=\phi\left(y_{t-1}-y_{t-2}\right)+\varepsilon_{t} \\
\hat{\phi}=\frac{\sum_{t=1}^{T}\left(y_{t}-y_{t-1}\right)\left(y_{t-1}-y_{t-2}\right)}{\sum_{t=1}^{T}\left(y_{t-1}-y_{t-2}\right)^{2}}
\end{gathered}
$$


When $c \neq 0$, equation (11) gives

$$
\left(\begin{array}{cc}
n & \sum_{t=1}^{T} \Delta y_{t-1} \\
\sum_{t=1}^{T} \Delta y_{t-1} & \sum_{t=1}^{T} \Delta y_{t-1}^{2}
\end{array}\right)\left(\begin{array}{l}
c \\
\phi
\end{array}\right)=\left(\begin{array}{c}
\sum_{t=1}^{T} \Delta y_{t} \\
\sum_{t=1}^{T} \Delta y_{t} \Delta y_{t-1}
\end{array}\right)
$$

Using Crammer's rule, results in

where

$$
\hat{c}=\frac{\nabla_{1}}{\nabla} \text { and } \hat{\phi}=\frac{\nabla_{2}}{\nabla}
$$

$$
\begin{gathered}
\nabla=n \sum_{t=1}^{T} \Delta y_{t-1}{ }^{2}-\left[\sum_{t=1}^{T} \Delta y_{t-1}\right]^{2} \\
\nabla_{1}=\left[\sum_{t=1}^{T} \Delta y_{t}\right]\left[\sum_{t=1}^{T} \Delta y_{t-1}^{2}\right]-\left[\sum_{t=1}^{T} \Delta y_{t-1}\right]\left[\sum_{t=1}^{T} \Delta y_{t} \Delta y_{t-1}\right] \\
\nabla_{2}=n \sum_{t=1}^{T} \Delta y_{t} \Delta y_{t-1}-\left[\sum_{t=1}^{T} \Delta y_{t}\right]\left[\sum_{t=1}^{T} \Delta y_{t-1}\right]
\end{gathered}
$$

$\operatorname{ARIMA}(2,0,0)$

$$
y_{t}=c+\phi_{1} y_{t-1}+\phi_{2} y_{t-2}+\varepsilon_{t}
$$

If $c=0$, then

$$
\begin{aligned}
& \hat{\phi}_{1}=\frac{\sum_{t=1}^{T} y_{t} y_{t-1} \sum_{t=1}^{T} y_{t-2}{ }^{2}-\sum_{t=1}^{T} y_{t} y_{t-2} \sum_{t=1}^{T} y_{t-1} y_{t-2}}{\sum_{t=1}^{T} y_{t-1}{ }^{2} \sum_{t=1}^{T} y_{t-2}{ }^{2}-\left(\sum_{t=1}^{T} y_{t-1} y_{t-2}\right)^{2}} \\
& \hat{\phi}_{2}=\frac{\sum_{t=1}^{T} y_{t-1}^{2} \sum_{t=1}^{T} y_{t} y_{t-2}-\sum_{t=1}^{T} y_{t} y_{t-1} \sum_{t=1}^{T} y_{t-1} y_{t-2}}{\sum_{t=1}^{T} y_{t-1}{ }^{2} \sum_{t=1}^{T} y_{t-2}{ }^{2}-\left(\sum_{t=1}^{T} y_{t-1} y_{t-2}\right)^{2}}
\end{aligned}
$$

If $c \neq 0$, then

$$
\left(\begin{array}{ccc}
n & \sum_{t=1}^{T} y_{t-1} & \sum_{t=1}^{T} y_{t-2} \\
\sum_{t=1}^{T} y_{t-1} & \sum_{t=1}^{T} y_{t-1}{ }^{2} & \sum_{t=1}^{T} y_{t-1} y_{t-2} \\
\sum_{t=1}^{T} y_{t-2} & \sum_{t=1}^{T} y_{t-1} y_{t-2} & \sum_{t=1}^{T} y_{t-2}{ }^{2}
\end{array}\right)\left(\begin{array}{l}
c \\
\phi_{1} \\
\phi_{2}
\end{array}\right)
$$

$$
=\left(\begin{array}{c}
\sum_{t=1}^{T} y_{t} \\
\sum_{t=1}^{T} y_{t} y_{t-1} \\
\sum_{t=1}^{T} y_{t} y_{t-2}
\end{array}\right)
$$

Using Crammer's rule, $c=\frac{\nabla_{1}}{\nabla}, \phi_{1}=\frac{\nabla_{2}}{\nabla}$ and $\phi_{2}=\frac{\nabla_{3}}{\nabla}$ where

$$
\nabla=\left|\begin{array}{ccc}
n & \sum_{t=1}^{T} y_{t-1} & \sum_{t=1}^{T} y_{t-2} \\
\sum_{t=1}^{T} y_{t-1} & \sum_{t=1}^{T} y_{t-1}^{2} & \sum_{t=1}^{T} y_{t-1} y_{t-2} \\
\sum_{t=1}^{T} y_{t-2} & \sum_{t=1}^{T} y_{t-1} y_{t-2} & \sum_{t=1}^{T} y_{t-2}{ }^{2}
\end{array}\right|,
$$

$$
\nabla_{1}=\left|\begin{array}{ccc}
\sum_{t=1}^{T} y_{t} & \sum_{t=1}^{T} y_{t-1} & \sum_{t=1}^{T} y_{t-2} \\
\sum_{t=1}^{T} y_{t} y_{t-1} & \sum_{t=1}^{T} y_{t-1}{ }^{2} & \sum_{t=1}^{T} y_{t-1} y_{t-2} \\
\sum_{t=1}^{T} y_{t} y_{t-2} & \sum_{t=1}^{T} y_{t-1} y_{t-2} & \sum_{t=1}^{T} y_{t-2}{ }^{2}
\end{array}\right|,
$$




$$
\begin{aligned}
\nabla_{2} & =\left|\begin{array}{ccc}
n & \sum_{t=1}^{T} y_{t} & \sum_{t=1}^{T} y_{t-2} \\
\sum_{t=1}^{T} y_{t-1} & \sum_{t=1}^{T} y_{t} y_{t-1} & \sum_{t=1}^{T} y_{t-1} y_{t-2} \\
\sum_{t=1}^{T} y_{t-2} & \sum_{t=1}^{T} y_{t} y_{t-2} & \sum_{t=1}^{T} y_{t-2}^{2}
\end{array}\right|, \\
\text { and } & \nabla_{3}=\left|\begin{array}{ccc}
n & \sum_{t=1}^{T} y_{t-1} & \sum_{t=1}^{T} y_{t} \\
\sum_{t=1}^{T} y_{t-1} & \sum_{t=1}^{T} y_{t-1}^{2} & \sum_{t=1}^{T} y_{t} y_{t-1} \\
\sum_{t=1}^{T} y_{t-2} & \sum_{t=1}^{T} y_{t-1} y_{t-2} & \sum_{t=1}^{T} y_{t} y_{t-2}
\end{array}\right| .
\end{aligned}
$$

$\operatorname{ARIMA}(2,1,0)$

$$
\Delta y_{t}=c+\phi_{1} \Delta y_{t-1}+\phi_{2} \Delta y_{t-2}+\varepsilon_{t}
$$

when, $\quad c=0, \quad \Delta y_{t}=\phi_{1} \Delta y_{t-1}+\phi_{2} \Delta y_{t-2}+\varepsilon_{t}$ which is

$$
y_{t}-y_{t-1}=\phi_{1}\left(y_{t-1}-y_{t-2}\right)+\phi_{2}\left(y_{t-2}-y_{t-3}\right)+\varepsilon_{t} \text {. }
$$

The result is $\hat{\phi}_{1}=\frac{\nabla_{1}}{\nabla}$ and $\hat{\phi}_{2}=\frac{\nabla_{2}}{\nabla}$, where

$$
\begin{gathered}
\nabla=\sum_{t=1}^{T}\left(y_{t-1}-y_{t-2}\right)^{2} \sum_{t=1}^{T}\left(y_{t-2}-y_{t-3}\right)^{2} \\
-\left[\sum_{t=1}^{T}\left(y_{t-1}-y_{t-2}\right)\left(y_{t-2}-y_{t-3}\right)\right]^{2} \\
\nabla_{1}=\sum_{t=1}^{T}\left(y_{t}-y_{t-1}\right)\left(y_{t-1}-y_{t-2}\right) \sum_{t=1}^{T}\left(y_{t-2}-y_{t-3}\right)^{2} \\
-\sum_{t=1}^{T}\left(y_{t}-y_{t-1}\right)\left(y_{t-2}-y_{t-3}\right) \sum_{t=1}^{T}\left(y_{t-1}-y_{t-2}\right)\left(y_{t-2}-y_{t-3}\right)
\end{gathered}
$$

and

$$
\begin{aligned}
\nabla_{2}= & \sum_{t=1}^{T}\left(y_{t-1}-y_{t-2}\right)^{2} \sum_{t=1}^{T}\left(y_{t}-y_{t-1}\right)\left(y_{t-2}-y_{t-3}\right) \\
& -\left[\begin{array}{l}
\sum_{t=1}^{T}\left(y_{t}-y_{t-1}\right)\left(y_{t-1}-y_{t-2}\right) \\
* \sum_{t=1}^{T}\left(y_{t-1}-y_{t-2}\right)\left(y_{t-2}-y_{t-3}\right)
\end{array}\right]
\end{aligned}
$$

$\operatorname{ARIMA}(\mathrm{P}, 0,0)$

$$
y_{t}=c+\phi_{1} y_{t-1}+\phi_{2} y_{t-2}+\phi_{3} y_{t-3}+\ldots+\phi_{P} y_{t-P}+\varepsilon_{t}
$$

given $c=0, A \Psi=B$. That is

$$
\begin{aligned}
& \left(\begin{array}{ccccc}
\sum_{t=1}^{T} y_{t-1}{ }^{2} & \sum_{t=1}^{T} y_{t-2} y_{t-1} & \sum_{t=1}^{T} y_{t-3} y_{t-1} & \cdots & \sum_{t=1}^{T} y_{t-p} y_{t-1} \\
\vdots & & \vdots & \vdots \\
\sum_{t=1}^{T} y_{t-1} y_{t-p} & \sum_{t=1}^{T} y_{t-2} y_{t-p} & \sum_{t=1}^{T} y_{t-3} y_{t-p} & \cdots & \sum_{t=1}^{T} y_{t-p}{ }^{2}
\end{array}\right)\left(\begin{array}{c}
\phi_{1} \\
\vdots \\
\phi_{p}
\end{array}\right) \\
& =\left(\begin{array}{c}
\sum_{t=1}^{T} y_{t} y_{t-1} \\
\vdots \\
\sum_{t=1}^{T} y_{t} y_{t-p}
\end{array}\right)
\end{aligned}
$$

where $\mathrm{A}$ is $p \times p$ matrix and $\Psi$ is a column matrix, that is $\Psi=\left(\begin{array}{llll}\phi_{1} & \phi_{2} & \phi_{3} \ldots \phi_{p}\end{array}\right)^{\prime}$. B is a column matrix:

$$
\left(\begin{array}{c}
\sum_{t=1}^{T} y_{t} y_{t-1} \\
\vdots \\
\sum_{t=1}^{T} y_{t} y_{t-p}
\end{array}\right)
$$

The expression for each parameter $\phi_{i}, i=1,2, \ldots, p$ can thus be determined using Crammer's rule or the Gauss-Schidel method. 
Given $\quad c \neq 0, A \Psi=B, \quad$ where $\Psi=\left(\begin{array}{llll}c, \phi_{1} & \phi_{2} & \phi_{3} \ldots \phi_{p}\end{array}\right)^{\prime}$. That is,

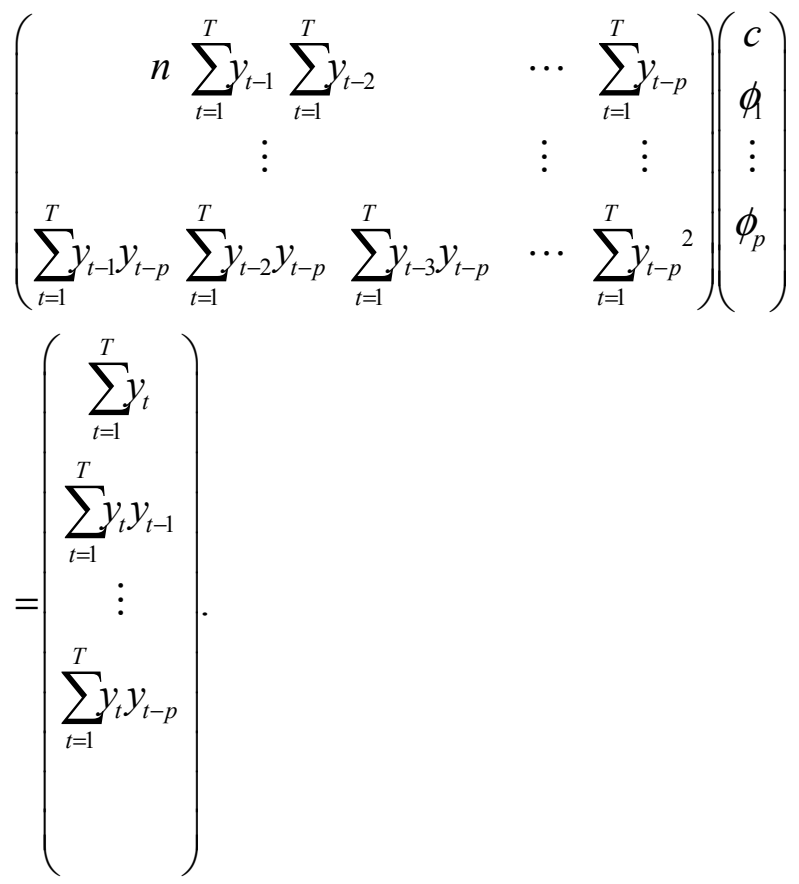

ARIMA $(P, 1,0)$

$$
\begin{aligned}
\Delta y_{t}= & c+\phi_{1} \Delta y_{t-1}+\phi_{2} \Delta y_{t-2} \\
& +\phi_{3} \Delta y_{t-3}+\ldots+\phi_{P} \Delta y_{t-P}+\varepsilon_{t}
\end{aligned}
$$

For the estimate of parameters in ARIMA $(p, 0,0)$ and $(p, 1,0)$, it is deduced that every term $y_{t-j}$ in ARIMA $(\mathrm{p}, 0,0)$ is replaced by $y_{t-j}-y_{t-j-1}$ in ARIMA $(\mathrm{p}, 1,0)$. Also, $\Psi$ is a $p$ column matrix for $c=0$, and $\Psi$ is a $(p+1)$ column matrix for $c \neq 0$.

\section{Results}

Daily temperature data for the maximum daily temperature of Ondo, Nigeria and Zaira, Nigeria from January 1995 to November 2005 are used in this study. Stationarity of a series is determined by the use of a correlogram for describing both autocorrelation and partial autocorrelation functions for the series. The series is non-stationary, it is therefore differenced once (i.e., $d=1$ ) to ensure stationarity. Figures 1 and 2 show the correlograms for the series after differencing each once (stationary at $d=1$ ). Also, the residual terms (white noise process or innovation series (Bobba, et al., 2006)) are independently and identically distributed because the autocorrelation function at various lags hover around zero (see Figure 1) (Gujarati, 2004). Similarly, Figures $3 a$ and $3 b$ show that residuals are normally distributed, thus, $\varepsilon_{t} \sim \operatorname{iid} N\left(0, \sigma^{2}\right)$.

When, $c=0$ (see Formula 1).

Formula 1: $\operatorname{ARIMA~}(\mathrm{P}, 1,0)$ when $c=0$

$$
\begin{aligned}
& \left(\begin{array}{cccc}
\sum_{t=1}^{T}\left(y_{t-1}-y_{t-2}\right)^{2} & \sum_{t=1}^{T}\left(y_{t-1}-y_{t-2}\right)\left(y_{t-2}-y_{t-3}\right) & \cdots & \sum_{t=1}^{T}\left(y_{t-1}-y_{t-2}\right)\left(y_{t-p}-y_{t-p-1}\right) \\
\vdots & \vdots & \vdots \\
\sum_{t=1}^{T}\left(y_{t-1}-y_{t-2}\right)\left(y_{t-p}-y_{t-p-1}\right) \sum_{t=1}^{T}\left(y_{t-2}-y_{t-3}\right)\left(y_{t-p}-y_{t-p-1}\right) & \cdots & \sum_{t=1}^{T}\left(y_{t-p}-y_{t-p-1}\right)^{2}
\end{array}\right)\left(\begin{array}{c}
\phi_{1} \\
\vdots \\
\phi_{p}
\end{array}\right) \\
& =\left(\begin{array}{c}
\sum_{t=1}^{T}\left(y_{t}-y_{t-1}\right)\left(y_{t-1}-y_{t-2}\right) \\
\vdots \\
\sum_{t=1}^{T}\left(y_{t}-y_{t-1}\right)\left(y_{t-p}-y_{t-p-1}\right)
\end{array}\right)
\end{aligned}
$$


Figure 1: Correlogram after Difference for Ondo, Nigeria

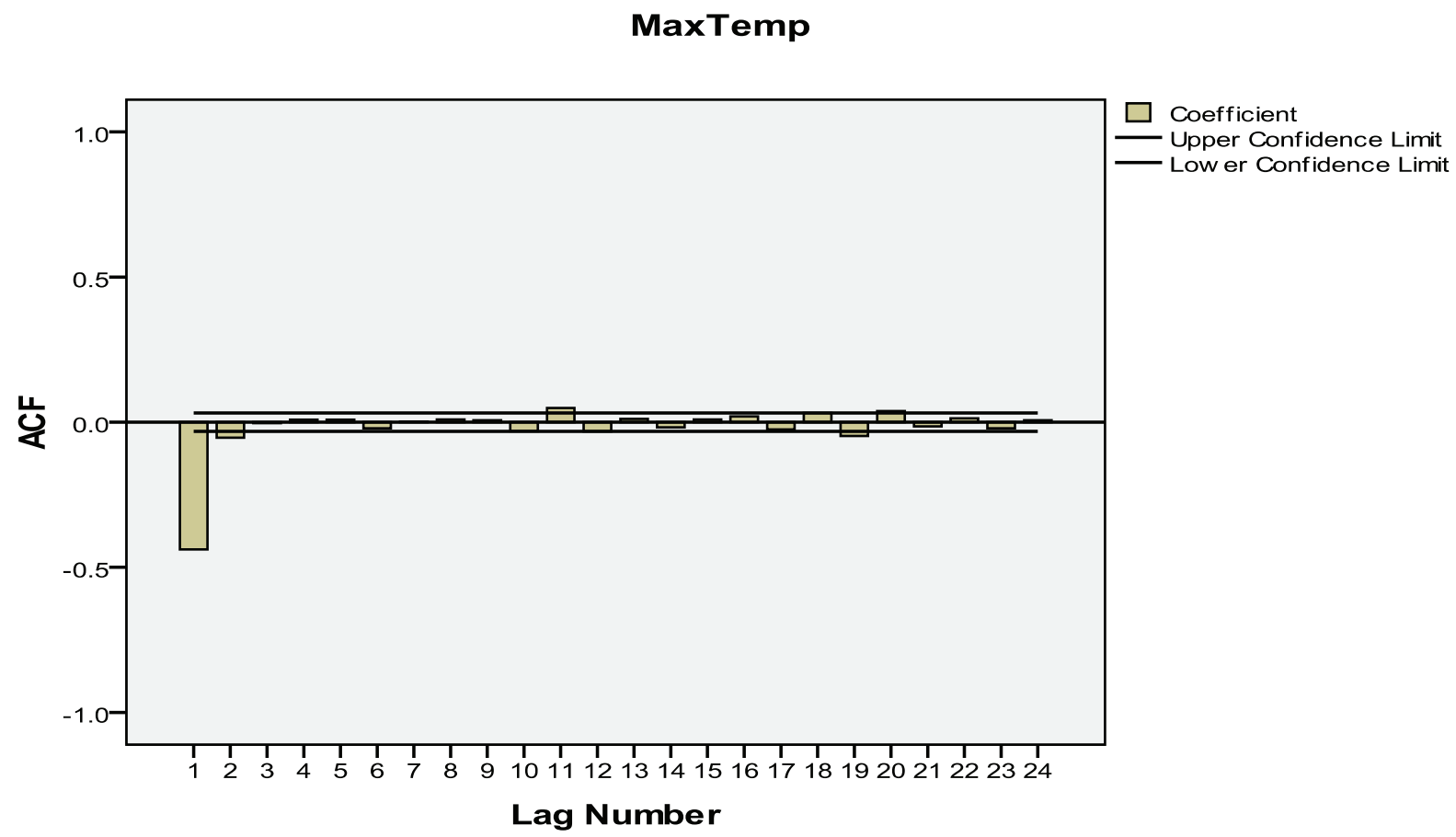

Figure 2: Correlogram after Difference for Zaira, Nigeria

\section{Temperature}

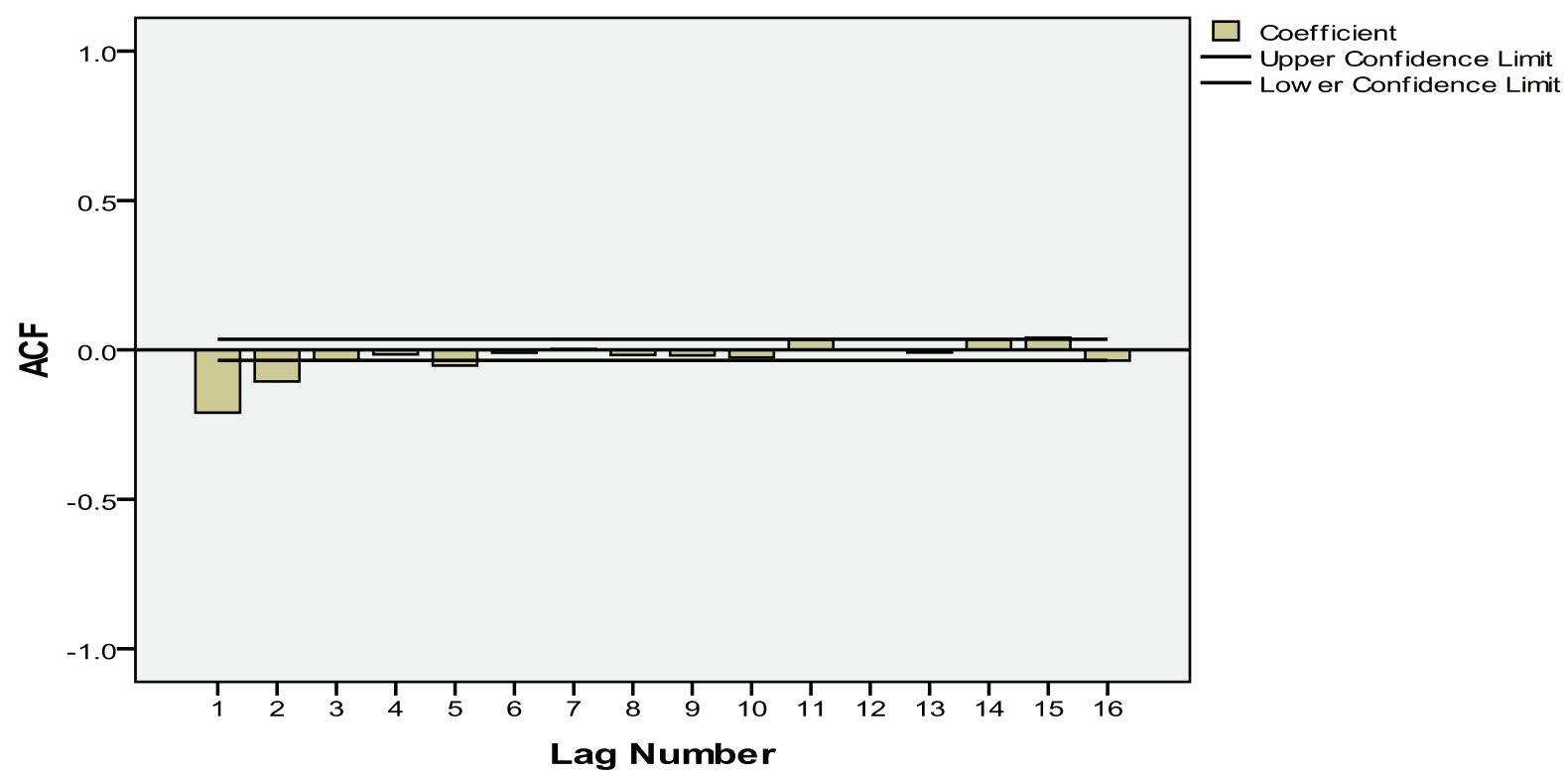


Figure 3(a): Histogram of Residuals for Ondo, Nigeria

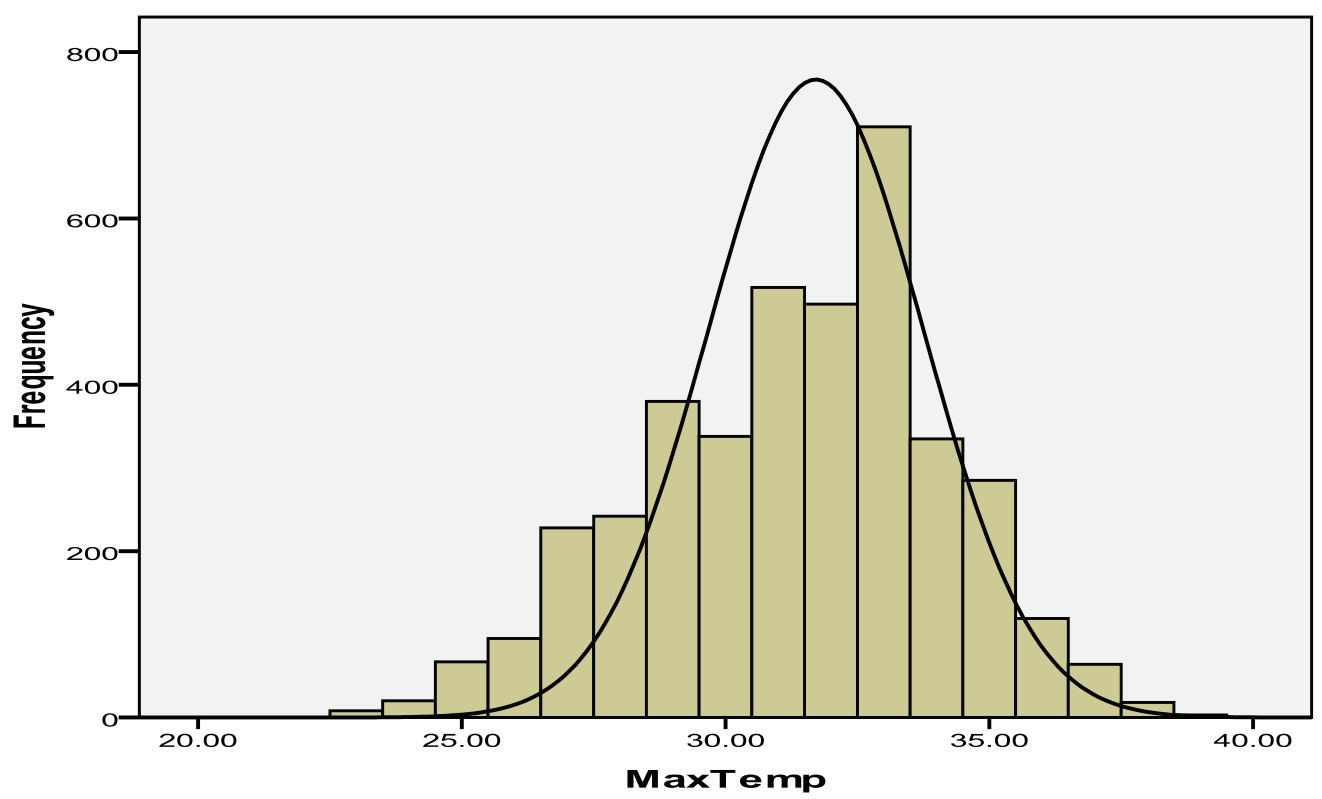

Figure 3(b): Histogram of Residuals for Zaira, Nigeria

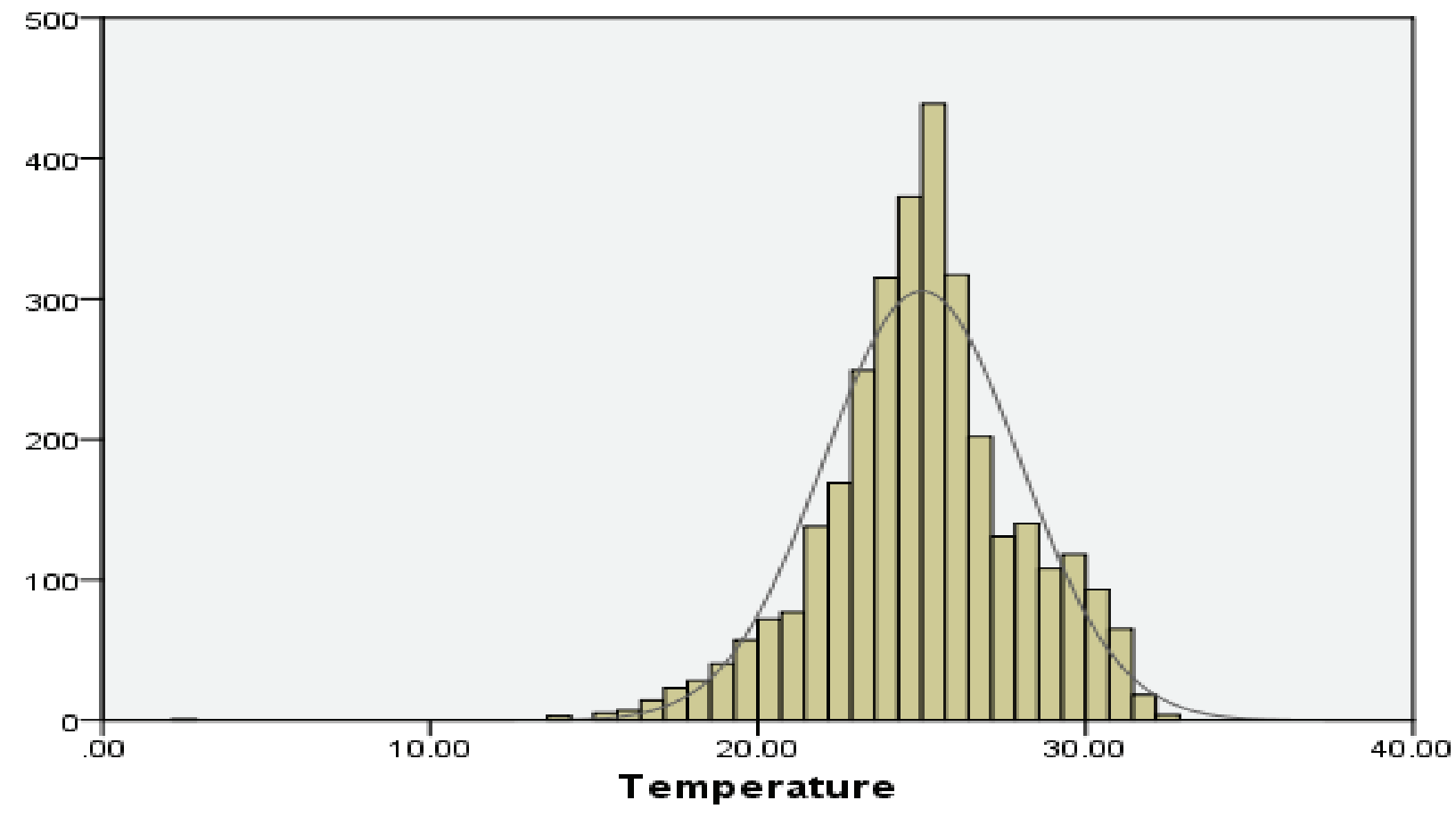


MAKINDE \& FASORANBAKU

Table 1: Various ARIMA Model Fits with Normalized BIC Values

\begin{tabular}{|c|c|c|c|c|c|c|c|c|}
\hline \multirow{2}{*}{$\begin{array}{l}\text { Model } \\
\text { Selection } \\
\text { Criteria }\end{array}$} & \multicolumn{8}{|c|}{ Normalized BIC Values for Model Fit } \\
\hline & $\begin{array}{c}\text { ARIMA } \\
(0,1,0)\end{array}$ & $\begin{array}{c}\text { ARIMA } \\
(0,1,1)\end{array}$ & $\begin{array}{c}\text { ARIMA } \\
(1,1,0)\end{array}$ & $\begin{array}{c}\text { ARIMA } \\
(1,1,1)\end{array}$ & $\begin{array}{c}\text { ARIMA } \\
(1,1,2)\end{array}$ & $\begin{array}{c}\text { ARIMA } \\
(2,1,0)\end{array}$ & $\begin{array}{c}\text { ARIMA } \\
(2,1,1)\end{array}$ & $\begin{array}{c}\text { ARIMA } \\
(2,1,2)\end{array}$ \\
\hline Ondo & 1.354 & 0.917 & 1.144 & 0.915 & 0.917 & 1.049 & 0.917 & 0.918 \\
\hline Zaira & 0.62 & 0.558 & 0.578 & 0.515 & 0.518 & 0.556 & 0.518 & 0.52 \\
\hline
\end{tabular}

Table 2: Adequacy Test Results of the Model

\begin{tabular}{|c|c|c|c|c|c|c|}
\hline \multicolumn{2}{|c|}{ Model Statistics } \\
\hline \multirow{2}{*}{ City } & \multirow{2}{*}{$\begin{array}{c}\text { Number of } \\
\text { Predictors }\end{array}$} & $\begin{array}{c}\text { Model Fit Statistics: } \\
\text { Stationary R-Squared }\end{array}$ & \multicolumn{2}{|c|}{ Ljung-Box Q(18) } & \multirow{2}{*}{$\begin{array}{c}\text { Number of } \\
\text { Outliers }\end{array}$} \\
\cline { 4 - 6 } Ondo & 0 & 0.105 & 28.999 & 16 & .024 & 0 \\
\hline Zaira & 0 & 0.105 & 28.999 & 16 & .024 & \\
\hline
\end{tabular}

Table 3: Parameter Estimates

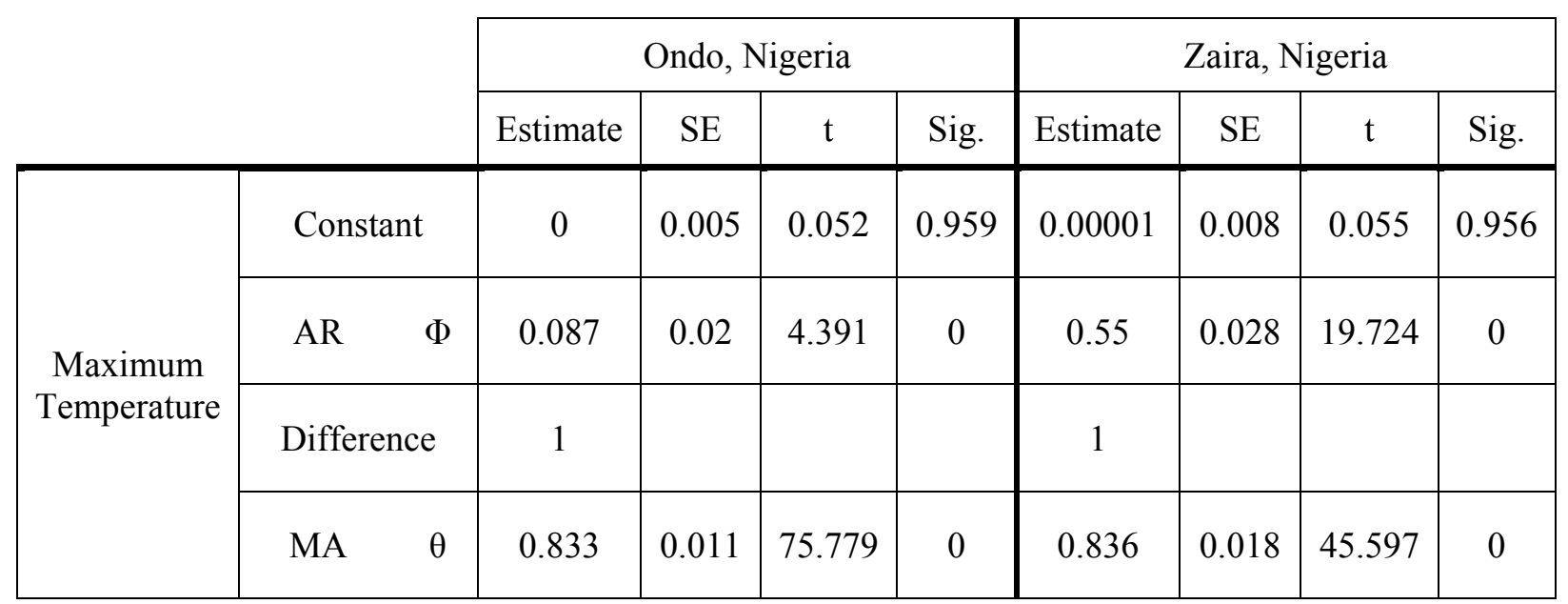




\section{IDENTIFICATION OF OPTIMAL ARIMA MODEL ON TEMPERATURE DATA}

Using a normalized BIC as the model selection criterion (that is, to test for goodness of fit) for various values of $p$ and $q$ (see Table 1), ARIMA $(1,1,1)$ has the least normalized BIC value, which equals 0.915 for Ondo and 0.515 for Zaira. Hence, the ARIMA $(1,1,1)$ is considered the best model for the maximum daily temperature data. for both Ondo and Zaira.

To test for the adequacy of the model, the Ljung-Box Statistic is used to test the randomness of residuals. The $p$-values of the Ljung-Box Statistic at various lags (in ACF and PACF) are less than 0.05; this shows that the data are random. The Ljung-Box Statistic for the model is 28.999 with a $p$-value $=0.024$, this establishes that the model is adequate (see Table 2).

Table 3 presents the estimates of parameters of the $\operatorname{ARIMA}(1,1,1)$ model for Ondo and Zaira. The model for Ondo is:

$$
y_{t}=0.00001+0.087 y_{t-1}+\varepsilon_{t}+0.836 \varepsilon_{t-1} .
$$

that is, $c=0.00001, \quad \phi=0.550, \quad$ and $\theta=0.836$ (see Table 5). Also, in testing for significance of the parameter estimates, Table 3 shows the t-statistics for the parameter estimates of the model. It is shown that a $c$ with $t=0.052$ and a $p$-value $=0.959$ is not significantly different from zero; thus, $c$ is redundant.

To improve the model result, $c$ was removed because it is redundant. This removal had no effect on the estimates of other parameters or on the Ljung-Box value of the model; rather it results in a smaller normalized BIC value (=0.912). Hence, the optimal model for maximum temperature of Ondo is:

$$
y_{t}=0.087 y_{t-1}+\varepsilon_{t}+0.833 \varepsilon_{t-1} .
$$

The invertibility behavior of the optimal model for Ondo, Nigeria is $\varepsilon_{t}=\sum_{i=0}^{\infty} \pi_{i} y_{t-i}$, because $c$ is redundant, where

$$
\pi_{i}= \begin{cases}1, & i=0 \\ -(1.920), & i=1 \\ (-1)^{i}\left[0.920\left(0.833^{i-1}+0.833^{i-2}\right)\right], & i=2,3,4, \ldots\end{cases}
$$

The model for Zaira is

$$
y_{t}=0.00001+0.550 y_{t-1}+\varepsilon_{t}+0.836 \varepsilon_{t-1},
$$

that is, $c=0.00001, \quad \phi=0.550, \quad$ and $\theta=0.836$ (see Table 3). Also, in testing for significance of the estimates of parameters, results show that a $c$ with $t=0.055$ and a $p$ value $=0.956$ is not significantly different from zero (see Table 3). Hence, $c$ is redundant.

To improve the model result, $c$ was removed; this had no effect on the estimates of other parameters or on the Ljung-Box value of the model, instead, it results in a smaller normalized BIC value $(=0.512)$. Hence, the optimal model for the maximum temperature of Ondo is:

$$
y_{t}=0.550 y_{t-1}+\varepsilon_{t}+0.836 \varepsilon_{t-1} \text {. }
$$

The invertibility behavior of the optimal model for Zaira is $\varepsilon_{t}=\sum_{i=0}^{\infty} \pi_{i} y_{t-i}$, because $c$ is redundant, where

$$
\pi_{i}= \begin{cases}1, & i=0 \\ -(2.386), & i=1 \\ (-1)^{i}\left[1.386\left(0.836^{i-1}+0.836^{i-2}\right)\right], & i=2,3,4, \ldots\end{cases}
$$

\section{Conclusion}

Autoregressive Integrated Moving Average (ARIMA) processes of various orders are presented with the goal of identifying an optimal model from a class of models. ARIMA $(p, d, q)$ model is formulated for daily maximum temperature data of Ondo, Nigeria and Zaira, Nigeria from 1995 to 2005. A normalized Bayesian Information Criteria (BIC) is used to 
measure performance of the models. ARIMA ( 1 , $1,1)$ is optimal and adequate for modeling the daily maximum temperatures because it has the least normalized BIC, parameters of the model are estimated and the redundant variable is removed. The behavioral pattern of the optimal model for each of the cities is reported.

\section{References}

Bobba, A. G., Rudra, R. P., \& Diiwu, J. Y. (2006). A stochastic model for identification of trends in observed hydrological and meteorological data due to climate change in watersheds. Journal of Environmental Hydrology, 14(10), 1-11.

Dobre, I., \& Alexandru, A. A. (2008). Modeling unemployment rate using Box-Jenkins procedure. Journal of Applied Quantitative Methods, 3(2), 156-166.

Fasoranbaku, O. A. \& Makinde, O. S. (2011). Behavioural pattern of causality parameter of autoregressive moving average model. Journal of Nigerian Association of Mathematical Physics, 19, 583-590.

Gujarati, D. N. (2004). Basic econometrics, $4^{\text {th }}$ Ed. McGraw-Hill: The McGraw-Hill Companies.

Hamilton, J. D. (1994). Time series analysis. Princeton, NJ: Princeton University Press.

Ljung, G. M., \& Box, G. E. P. (1978). On a measure of a lack of fit in time series models. Biometrika, 65(2), 297-303

Lorenz, E. N. (1963). Deterministic nonperiodic flow. Journal of Atmospheric Science, 20, 130-141.
Makinde, O. S. (2011). Behavioural Pattern of Invertibility Parameter of ARIMA Model. Journal of Nigerian Association of Mathematical Physics, 19, 591-606.

Meko D. M. (1981) Application of Box Jenkins Methods of Time Series Analysis to reconstruction of drought from tree rings. Ph.D. Dissertation, University of Arizona.

Nelson, C. R. (1973) Applied time series analysis. San Francisco, CA: Holden-Day.

Ojo, J. F. (2008). Identification of optimal models in higher order of integrated autoregressive models and autoregressive integrated moving average models in the presence of $2^{\mathrm{k}}-1$ subsets. Journal of Modern Mathematics and Statistics, 2(1), 7-11.

Ojo, J. F. (2009). On the estimation and performance of subset autoregressive integrated moving average models. European Journal of Scientific Research, 28(2), 287-293.

Schwarz, G. E. (1978). Estimating the dimension of a model. Annals of Statistics, 6(2), 461-464.

Shittu, O. I., \& Yaya, O. S. (2009). Measuring forecast performance of ARIMA and ARFIMA: An application to US Dollar/UK Pound foreign exchange rate. European Journal of Scientific Research, 32(2), 167-176.

Tuan, P. (1978). Establishment of parameters in the ARIMA model when the characteristic polynomial of MA operator has a unit zero. Annals of Statistics, 6(6), 1369-1389. 